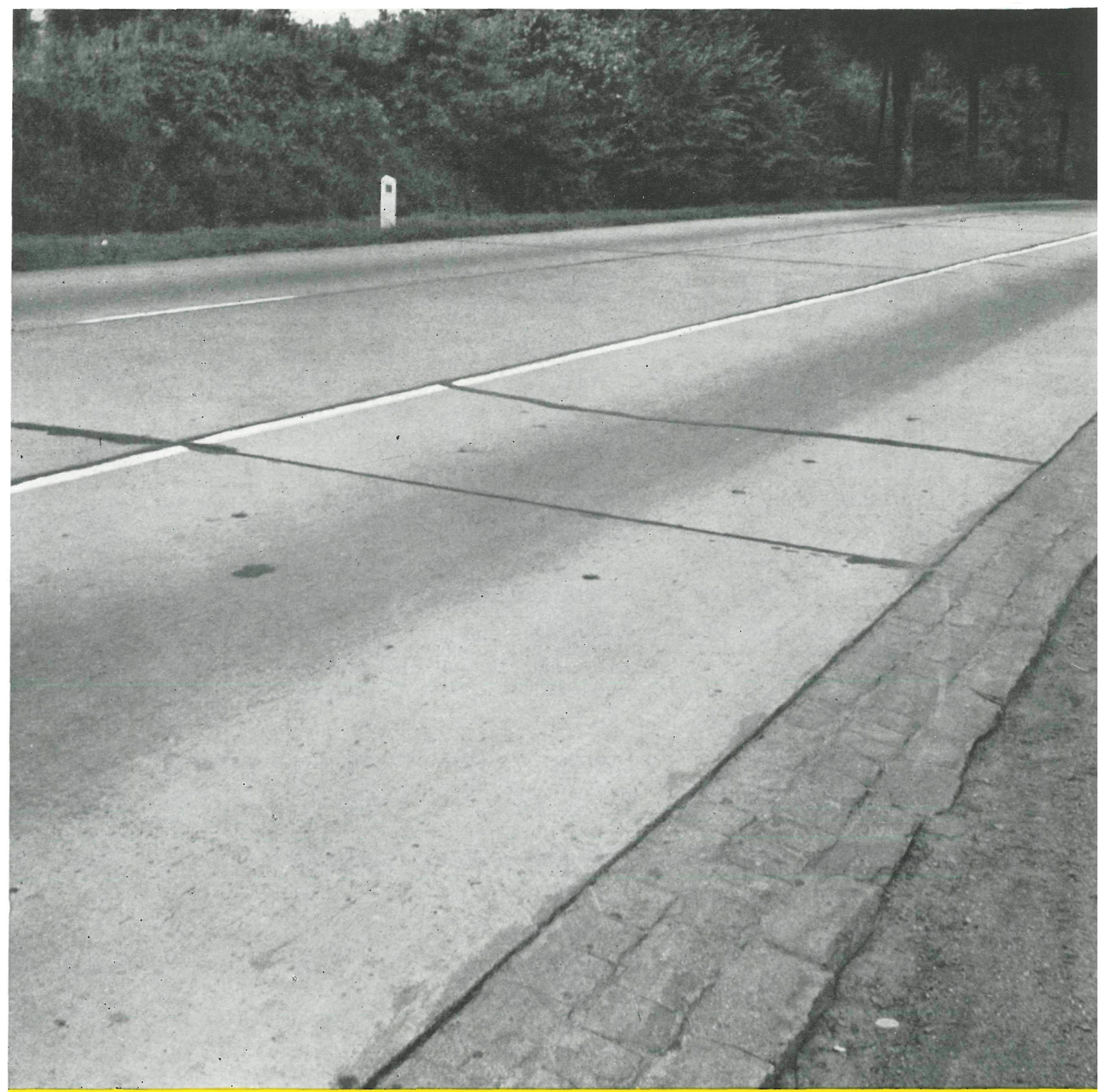

\title{
reparación de firmes de hormigón por inyección
}

\section{SANDRO ROCCl, ingeniero de caminos}

\section{$514-44$}

\section{Origen del problema}

Uno de los reproches que con más frecuencia se han formulado a los firmes de hormigón ha sido el de su difícil reparación en caso de avería, en contraste con el fácil y rápido "parcheado" que permiten los firmes flexibles. Esta facilidad depende tanto de su comportamiento estructural (en el firme flexible sólo trabaja la porción del mismo directamente afectada por las cargas) como de las propiedades adhesivas de los materiales empleados, de tipo bituminoso. Estas circunstancias nc concurren generalmente en los firmes rígidos de hormigón; por lo que el único remedio eficaz en caso de avería era la reconstrucción total de las losas afectadas previa su demolición. Esto es fundamentalmente antieconómico, salvo casos especiales.

El proceso de la avería en el firme de hormigón es hoy sobradamente conocido, de modo que la técnica puede poner un remedio eficaz a sus efectos, atacando directamente las causas. Entre éstas, la principal, y casi única, es el fallo del subsuelo, generalmente en presencia del agua. 
A ésta se pueden reducir las demás causas, pues el efecto de todas ellas es la fisura, que permite la entrada del agua bajo el firme, perjudicando, y aun anulando, las cualidades sustentantes del terreno o capa de base. He aquí el porqué las juntas que por varias razones el proyectista se ve obligado a disponer en el firme rígido, constituyen puntos débiles a través de los cuales puede penetrar el agua y disminuir la resistencia del terreno subyacente, sometiendo a la acción perjudicial de las heladas y provocando, incluso, con ciertos suelos finos, el fenómeno del "bombeo" o vaciado parcial por efecto de las cargas.

Todas estas causas (que se reducen, como hemos visto a una sola: la posibilidad de penetrar el agua bajo el firme y comprometer la resistencia del terreno o el apoyo de la losa) tienen determinados efectos, según su gravedad. Desde el asiento diferencial de losas contiguas hasta la fisuración y ulterior fragmentación de las losas afectadas, existe toda una serie de averías, que no sólo molesta al tráfico, sino que se agravan por la acción del mismo, estableciéndose un ciclo de realimentación o círculo vicioso. Como inicialmente (salvo errores de proyecto siempre imprevisibles) no existen grietas, el círculo vicioso se suele iniciar por las juntas. De aquí que la técnica moderna tienda a reducirlas tanto en cantidad como en importancia.

Cabe replicar a la objeción de que es muy difícil reparar un firme de hormigón averiado, con dos observaciones. La primera es que la avería del firme de hormigón es menos "escandalosa" que el bache clásico de los firmes flexibles e, incluso, menos perjudicial para el tránsito rodado, debido a su menor "dimensión" vertical. Una fisura puede ser detectada y evitarse su progreso mucho antes de que constituya un peligro para la circulación; en un firme flexible, el bache primero se produce y después se repara. Pero es que, además, la técnica, tras un primer período de vacilaciones debidas al desconocimiento del comportamiento de determinados materiales, ha sabido encontrar hoy una respuesta satisfactoria al problema de la reparación rápida, cómoda y eficaz de un firme de hormigón, tal y como anteriormente se hizo en el caso del firme flexible.

En lo que sigue, pues, me ocuparé esquemáticamente del problema básico del desnivelamiento de los labios de una junta, causado por el asiento diferencial de las losas adyacentes. Se trata de solucionar el problema en esta etapa, antes que tome mayor importancia. Extenderé el tema hasta tratar del caso en que, por haberse quedado vacía la parte subyacente a la junta, el extremo de la losa (casi siempre la de "aguas abajo", si se permite el símil hidráulico) trabaja en voladizo, y se produzca una fisura transversal característica, paralela a la junta y a unos $2 \mathrm{~m}$ de ella.

En la fase de proyecto se pueden prever y evitar en parte estos desperfectos, por medio de los siguientes criterios:

a) Supresión total o, al menos, distanciamiento, de las juntas de dilatación, que por su importante discontinuidad marcan un punto más dẻbil que el de las juntas de contracción.

b) Provisión de una capa de base de resistencia y estabilidad suficiente.

c) Establecimiento de una interacción entre las losas contiguas a la junta, por medio de dispositivos de transferencia de cargas.

En firmes ya construídos y en los que, debido al incesante aumento de las cargas del tráfico, se haya producido una desnivelación y aun fisuración de las zonas próximas a una junta, caben las siguientes soluciones:

1) Parcheado con un firme bituminoso que absorba la desnivelación. Este procedimiento, aparte del evidente perjuicio estético (no despreciable en los firmes de hormigón, en los que las reparaciones de este tipo son escandalosamente ostensibles), parece muy comprometido a la adherencia entre el hormigón y el producto bituminoso, que no siempre resulta como sería de desear.

2) Levantamiento y reconstrucción de la losa afectada. Este antieconómico procedimiento es obligado castigo del descuido del firme, que ha consentido su deterioro hasta extremos tales que impiden cualquier otro procedimiento de reparación.

3) Superposición de atra losa de hormigón. Este procedimiento, descrito ya en INFORMES número 126, es aplicable en determinados casos en vez del anterior, siendo más ventajoso que éste, pues se ahorran el levantamiento y consolidación del terreno, amén del menor espesor de flrme nuevo necesario.

4) Parcheado con mortero. Como el primer procedimiento exige una adherencia perfecta entre el parche y la losa vieja, y no es aplicable en caso de fisuras que se transmitirían a través del parche; pero tiene una ventaja estética innegable, aparte de la uniformidad de rodadura conseguida.

5) Inyección bajo la losa de un mortero de cemento y arena, que reconstituye el terreno perdido subyacente dejándola de nuevo al nivel anterior. Bien llevado, este procedimiento es el mejor de todos los expuestos cuando la losa está muy fisurada.

En este artículo trataré de exponer con más detalle este último procedimiento de reparación por inyección:

Consiste en inyectar-como su nombre indica-bajo la losa averiada por desnivelación por asiento y aun pérdida de contacto con el suelo, una mezcla adecuada, a través de orificios practicados al efecto en la losa.

Existe experiencia de esta clase de trabajos en Estados Unidos, Holanda y Bélgica; el resultado ha sido, generalmente, muy satisfactorio, bajo condiciones de tráfico muy variadas. Cada país sigue una técnica ligeramente diferente, y voy a procurar tanto resumirlas como enjuiciarlas. 

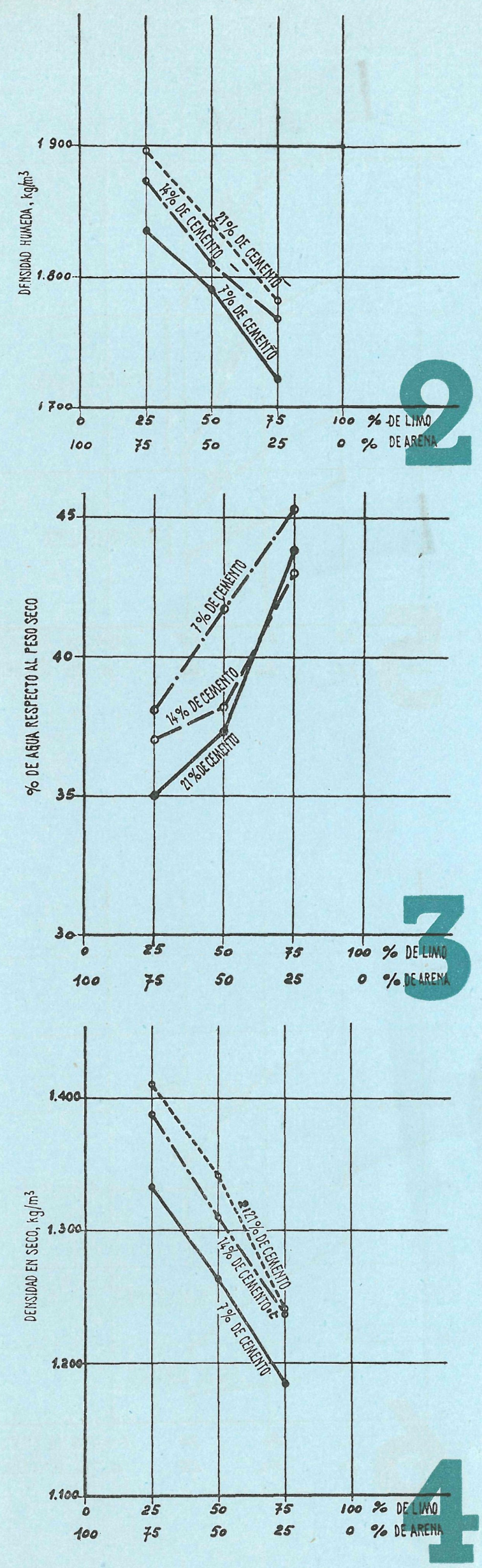


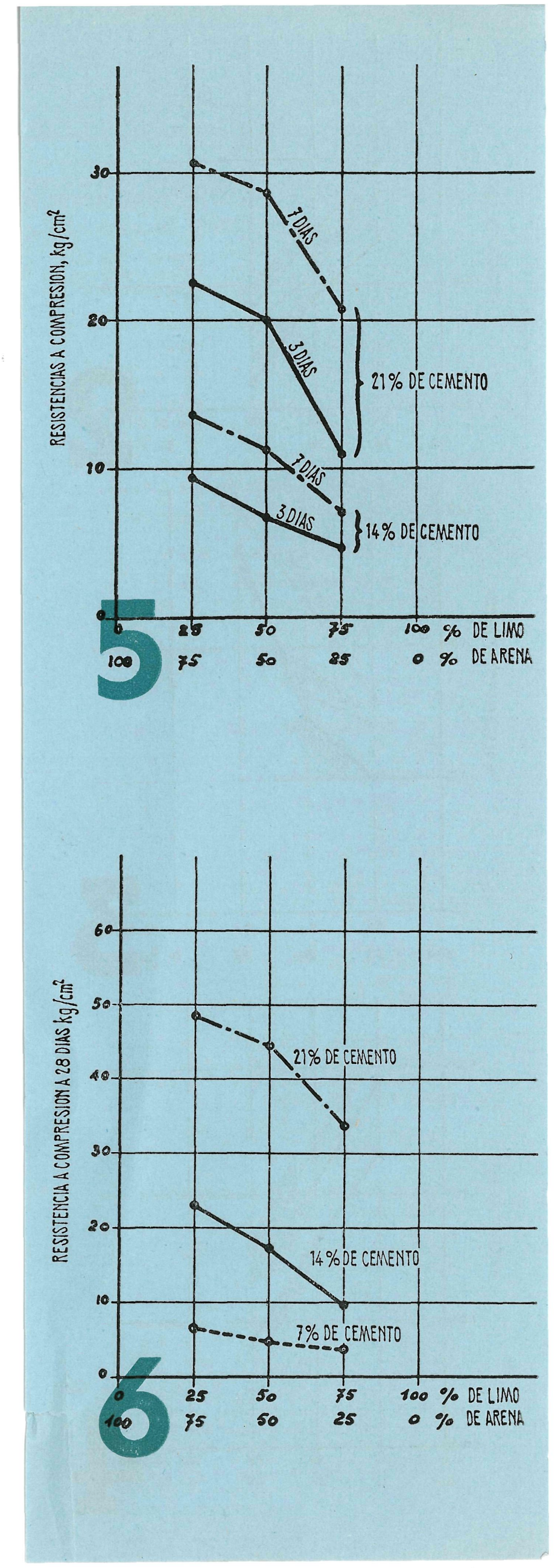




\section{Caraeteristicas de la mezcla inyectada}

Los componentes son arena, limo y cemento amasados con agua hasta obtener una consistencia flúida dispersa (aunque no floculada).

La consistencia adecuada exige una alta proporción de finos (constituídos por el limo y el cemento); sin embargo, el limo reduce la resistencia, por lo que debe limitarse su cantidad.

En Holanda se prescribe la siguiente mezcla:

\begin{tabular}{lr} 
Arena $<0,5 \mathrm{~mm}$ & 60 a $80 \%$ peso seco \\
Limo $<0,05 \mathrm{~mm}$ & 33 a $13 \%$ peso seco \\
Cemento & $7 \%$ peso seco \\
\cline { 2 - 2 } & \\
Agua & $100 \%$ peso seco total \\
\cline { 2 - 3 } &
\end{tabular}

Por el contrario, en los Estados Unidos se presentan las siguientes proporciones en volumen:

$\begin{array}{lr}\text { Arena } & 15 \text { a } 42 \% \text { volumen seco } \\ \text { Limo } & 30 \text { a } 63 \% \text { volumen seco } \\ \text { Cemento } & 16 \text { a } 40 \% \text { volumen seco } \\ \text { Agua } & \simeq 50 \% \text { volumen seco total }\end{array}$

La experimentación llevada a cabo en Bélgica en el año 1958 por el Centre d'Information de l'Industrie Cimentière Belge, con diversas proporciones de arena, cemento y limo, arrojan los siguientes resultados recomendables a igualdad de consistencia:

1. El porcentaje de agua aumenta si aumenta el contenido de limo.

2. Iguaimente aumenta el agua necesaria si disminuye la cantidad de cemento. Esto es corriente, aunque aparentemente contradictorio, para conservar la consistencia en mezclas pobres.

3. El mantener bajo el contenido de agua, sobre todo con bajas proporciones de cemento, disminuye la retracción de la mezcla $\mathrm{y}$, por tanto, el asiento de la inyección.

4. La compacidad y resistencia de la mezcla aumenta con el contenido de cemento y con la relación arena/limo.

5. El tiempo de fraguado aumenta por bajas proporciones de cemento.

A la vista de estos resultados experimentales, recogidos en las figuras números 2 a 6 , se deducen las siguientes recomendaciones:

1. La proporción de elementos $>0,3 \mathrm{~mm}$ deben ser $<10 \%$.

2. La relación arena/limo debe ser $>2$, aumentándose este valor hasta 5 ó 6 en caso de arenas muy finas, limos arcillosos o mayor proporción de cementos; es decir, al aumentar los finos.

3. La baja proporción de cemento $(7 \%)$ no es económica; vale más aumentarla a 15 ó $20 \%$, haciendo un verdadero suelo-cemento.

4. La cantidad de agua debe ser la mínima compatible con una fácil inyección; en general, alrededor del $40 \%$ del volumen seco.

Resumiendo, se prescriben las dosificaciones arena-limo holandesas, junto con el contenido de cemento americano, es decir:

Arena (módulo de finura $<1$ ): 55 a $75 \%$ volumen seco.

Limo $(<0,05 \mathrm{~mm}): 30$ a $10 \%$ volumen seco.

Cemento: $15 \%$ volumen seco. Agua: $40 \%$ del total.

Llamaré la atención sobre el hecho de que la dosificación se estipula referida al volumen seco de los áridos; como éstos se encuentran húmedos en la obra, habrá que reducir el porcentaje de agua incluso al $30 \%$; al mismo tiempo, el porcentaje de cemento respecto al árido seco se habrá elevado automáticamente (fig. 7).

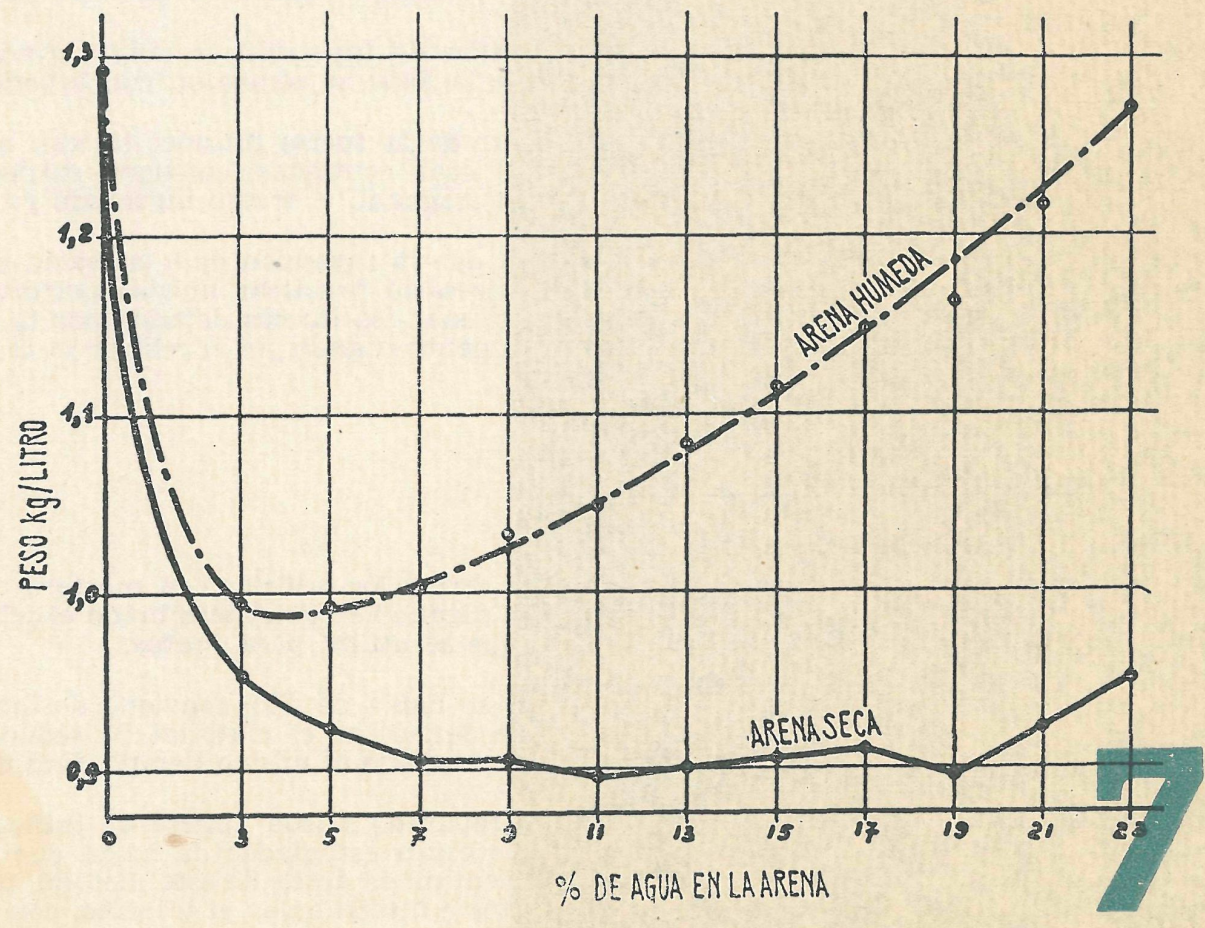




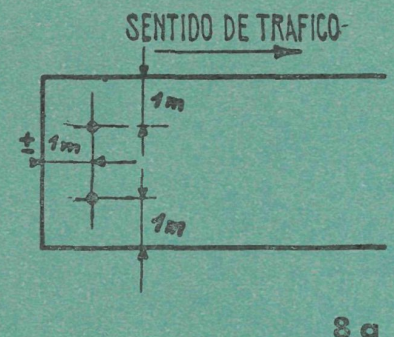

$8 a$

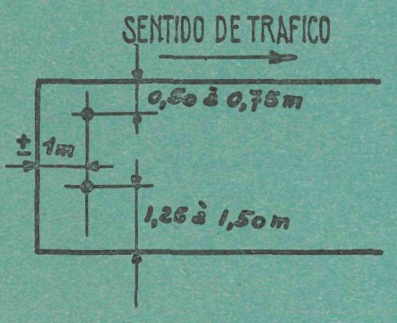

$8 b$

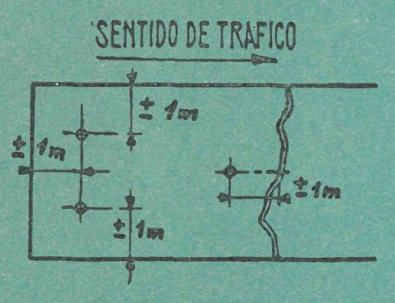

$8 c$

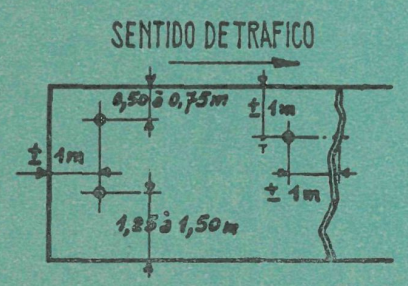

$8 \mathrm{~d}$

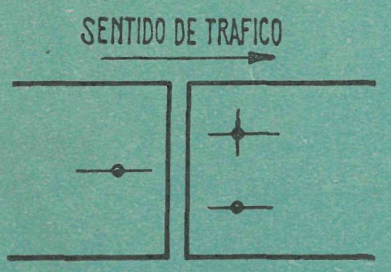

8 e

\section{Disposición de la inyección}

Los orificios se practican en la losa averiada por medio de una sonda especial. Su diámetro suele ser de 5 centímetros.

Aunque cada caso constituye un problema independiente se pueden dar algunas reglas sobre la disposición de los orificios que se van a inyectar.

En general, la losa asentada "aguas abajo" presenta un desnivel uniforme con la contigua. Entonces, dos orificios laterales, situados a $1 \mathrm{~m}$ de cada borde, pueden bastar si la losa no está agrietada o la fisura dista unos $2 \mathrm{~m}$ de lá junta; si la fisura está a $3 \mathrm{~m}$ ó más, se necesita un tercer orificio central a $1 \mathrm{~m}$ de la fisura. El objeto de este último es de colmatar los huecos que deje la losa al levantarse por efecto de los dos primeros, evitando que trabaje como viga apoyada.

Si la desnivelación es importante (>15 mm) habrán de acercarse más los orificios a los costados de la losa. Si la desnivelación no es uniforme, aumentando hacia la junta longitudinal, habrán de correrse todos los orificios hacia dicha junta (fig. 8).

Si también la losa "aguas arriba" ha asentado, en general basta con un solo orificio central.

\section{Técnica operaforia}

Una vez abiertos los orificios, primero se procede a inyectar aire comprimido, para despejar el camino a la inyección, y sobre todo eliminar el agua infiltrada y evitar la dilución de la mezcla. Este soplado es más eficaz si se realiza "a golpes", abriendo y cerrando bruscamente el paso del aire.

Los componentes de la mezcla, dosificados y pasados por un tamiz de $4 \mathrm{~mm}$, se amasan en una hormigonera pequeña, y luego se pasan al depósito de la inyectadora, de tipo corriente, que la envia a los orificios, empezando por el más bajo.

Es importante proceder lentamente y por pasadas sucesivas, a fin de evitar apoyos localizados que den lugar a fisuras; la operación puede terminarse cuando la losa se halle a nivel, o mejor, un poco más alta que la contigua (para compensar el asiento posterior). Una prueba del buen reparto de la inyección la constituye el aparecer la mezcla por los otros agujeros, los cuales, para evitar pérdidas, se pueden tapar con clavijas de madera.

Si la presión de inyección es muy elevada, pueden producirse fugas por los bordes de la losa; se combaten con tapones de arcilla bien apisonada.

Si, a pesar de la fuerte presión, la losa no se levanta más, so peligro de levantar las losas contiguas, conviene suspender la operación y reanudarla cuando haya fraguado el tramo inyectado ya (generalmente 24 horas).

A medida que la inyección deje en vacío partes más considerables de losa, puede ser necesario practicar nuevas perforaciones a lo largo del eje de la losa, para rellenar los huecos dejados por la losa al elevarse. Se puede pasar al orificio siguiente cuando por él refluye ya la mezcla inyectada por el anterior.

\section{Acabado}

Se deben cerrar los orificios con mortero rico de cemento, cuidadosamente compactado. Alguna vez se ha terminado el sellado con un producto bituminoso análogo al que se utiliza para juntas.

Y ya que se habla de ello, conviene destacar que la operación de levantamiento suele deteriorar el material de sellado de juntas; por lo que resulta conveniente renovarlo al mismo tiempo, tras una cuidadosa limpieza del surco.

El firme reparado puede abrirse al tráfico al cabo de 24 horas, pues las dosificaciones están estudiadas de modo que su fraguado acabe antes de ese plazo. Esta ventaja es única de este método, pues todos los demás enumerados son más lentos e interrumpen el tránsito, con la consiguiente molestia. 